\title{
Discussion on Liver Preoperative Planning System Based on Deep Learning
}

\author{
Xuehu Wang ${ }^{1,2 *}$, Jie Zhao ${ }^{1,2}$, Ying Ye ${ }^{1}$, Zihan Guo ${ }^{1}$, Yongchang Zheng ${ }^{3}$ and Shuaiqi Liu ${ }^{1,2}$ \\ ${ }^{1}$ School of Electronic and Information Engineering, Hebei University, Baoding, Hebei 071000, P. R. China,
}

${ }^{2}$ Key Laboratory of Digital Medical Engineering of Hebei Province, Baoding, Hebei 071000, P. R. China

${ }^{3}$ Department of Liver Surgery, Peking Union Medical College Hospital, Chinese Academy of Medical Sciences and Peking Union Medical College (CAMS \& PUMC), Beijing 100010, P. R. China

Submission: September 05, 2018; Published: September 17, 2018

"Corresponding author: Xuehu Wang, School of Electronic and Information Engineering, Hebei University, Baoding, China, 071000,

Tel: 0312-5077365; Email: xuehuwang@163.com

\begin{abstract}
Liver cancer has become the second largest cancer death rate in the world, and the prevention and treatment of liver diseases is extremely urgent. It has grown up to be a hot spot and focus of the world. With the development of medical imaging and the introduction of "precise surgery", the development of preoperative planning system for liver diseases has become a research hotspot. Accurate preoperative planning system relies on the development of image processing technology, and the introduction and application of deep learning just improve the accuracy of image processing technology and promote the development process of liver preoperative planning system. This paper mainly expounds the main problems of today's deep learning-based liver preoperative planning system and discusses the solution to the problem and the development direction of the preoperative planning system.
\end{abstract}

Keywords: Liver; Preoperative planning system; Deep learning; Precise surgery

Abbreviations: CT: Computed Tomography; MRI: Magnetic Resonance Imaging; PET: Positron Emission Tomography; US: Ultrasound

\section{Introduction}

The liver is the largest digestive gland and detoxification organ in the human body, and it is also a place for bile production. The integration of many functions has made it one of the main organs that are prone to tumors. In recent years, liver cancer has become the second largest cancer death rate in the world. Therefore, prevention and treatment of liver diseases are urgently needed, and it has become a hot spot and focus of the world [1].

With the development of medical imaging, Computed Tomography (CT), Magnetic Resonance Imaging (MRI), Positron Emission Tomography (PET) and Ultrasound (Ultrasound) technology have become the main basis for the diagnosis and treatment of clinical liver diseases [2].

In the past two decades, medical images have evolved from simple anatomical imaging to blood flow, metabolism and functional imaging. The imaging speed is getting faster and faster, and the image resolution is getting higher and higher. The development of medical imaging from two-dimensional to three-dimensional and functional imaging has led to a significant increase in the accuracy of clinical disease diagnosis, which has prompted liver surgery to enter the era of "precise surgery."
"Accurate Surgery" requires preoperative planning before liver surgery, which encourages the planning of surgical procedures to avoid important organs such as intrahepatic vessels and adjacent organs, improve the success rate and efficiency of surgery, reduce the workload of doctors, and make more Patients receive timely and effective treatment.

The development of the preoperative planning system is inseparable from the precise image segmentation technology. By segmenting the liver, the shape and contour information of the liver and the structural information of the lesion can be detected from the medical image. The information is used for liver function evaluation, tumor recognition and surgical treatment. Etc. has important clinical application value. However, the liver is adjacent to the organ tissues such as the spleen, stomach and intestine, and the difference in gray scale characteristics between the organs and tissues is small. In addition, the individual differences of the liver are large, and the tissue structure and spatial position are susceptible to external forces. The deformation of the amplitude. So far, the automatic and accurate segmentation and detection of liver contours is still a worldwide problem [3]. 
The traditional image segmentation technology mainly includes two parts: feature extraction and classifier. The design complexity of the feature extraction algorithm, application limitations, stability, and the diversity of specific feature extraction algorithms combined with specific classifiers limit the development of image processing technology [4]. The emergence of deep learning algorithms makes it possible to solve the above limitations and provide new ideas for image processing. Deep learning is a new field in machine learning research. Its motivation lies in establishing and simulating the neural network for human brain analysis and learning. It mimics the mechanism of human brain to interpret data, and it is expected to improve the segmentation accuracy of segmentation liver [5].

\section{Discussion and Suggestion of Existing Problems}

\section{Strengthen cooperation between fields}

With the development of computer vision and artificial intelligence, the accuracy of medical image segmentation continues to rise, which has greatly helped medical diagnosis. However, artificial intelligence scientists do not understand clinical needs, and clinicians do not understand the specific techniques of artificial intelligence, which leads to artificial intelligence not meeting the clinical specific needs [6]. In order to promote the application of artificial intelligence in the medical field, extensive cooperation between clinicians and machine learning scientists should be strengthened. This collaboration will solve the problem that machine learning researchers cannot obtain medical data, and it can also help machine learning researchers to develop deep learning algorithms that are more in line with clinical needs and apply them to computer-aided diagnostic equipment to improve diagnostic efficiency and accuracy.

\section{Medical image data sharing}

The training of deep learning algorithms requires a large number of data sets. However, compared with natural images, annotated medical images are relatively scarce and costly to label. Due to the sensitivity and particularity of the medical field, the annotation of medical data requires a large number of experts to manually complete, which requires a lot of manpower and resources. However, even in this case, due to the complexity of the case and the ambiguity of the image itself, the labeling is not guaranteed to be completely accurate [7]. In order to overcome this problem, different medical service providers need to share data resources or establish a medical image knowledge base to provide more data support for artificial intelligence researchers.

\section{Development of deep learning technology}

Currently, most medical image segmentation uses supervised deep learning algorithms. However, for some rare diseases that lack large amounts of data support, supervised deep learning algorithms cannot perform their performance. In order to overcome the problem of lack of data or data unavailability, the study of deep learning algorithms can be transferred from the supervised field to the semi-supervised or unsupervised field [8]. How to effectively use semi-supervised and unsupervised algorithms in the medical field without affecting the accuracy of the medical system, which is a problem that many researchers are currently studying, but does not provide a clinically acceptable solution, which will be A direction that needs to be studied.

\section{Conclusion}

Deep learning algorithm has important theoretical significance and practical application value in the field of medical image processing. This paper looks forward to the future development of deep learning technology in the field of medical image processing from three aspects: strengthening exchanges and cooperation between fields, medical image data sharing, and deep learning technology. The development of deep learning algorithms will inevitably play a positive role in the development of medical image segmentation and liver preoperative planning systems. In short, with the trend of big data, combined with the potential of artificial intelligence, strive to make deep learning algorithms make greater progress in the medical field and realize the original intention of intelligent medical care at an early date, build a precise preoperative planning system, and improve the success rate of surgery.

\section{Acknowledgement}

This work was supported by the Beijing Natural Science Foundation (No. L172055), the Beijing Municipal Science \& Technology Commission Research Fund (No. Z171100000417004) and the China Postdoctoral Fund (No. 2018M631755). Natural Science Foundation of China under grant (No. 61401308) and (No. 61572063). Hebei University improve comprehensive strength special funds in the Midwest (No. 801260201011). China Postdoctoral Fund (No. 2018M631755). Hebei Province High-level Talent Funding Project - Post-doctoral Research Projects Selective Funding (No. B2018005002)

\section{References}

1. Wang X, Zheng Y, Gan L, Wang X, Sang X, et al. (2017) Liver segmentation from ct images using a sparse priori statistical shape model (SP-SSM). Plos One 12(10): e0185249.

2. Wang X, Yang J, Ai D, Zheng Y, Tang S, et al. (2015) Adaptive mesh expansion model (AMEM) for liver segmentation from ct image. Plos One 10(3): e0118064.

3. Zheng Y, Li H, Yang H, Xie F, Kang K, et al. (2018) Soft-tissuesegmentation methods during image-guided precision liver surgery. Gastroenterology Report 6(3): 239-241.

4. Beghdadi A, Larabi MC, Bouzerdoum A, Iftekharuddin KM (2013) A survey of perceptual image processing methods. Signal Processing Image Communication 28(8): 811-831.

5. Milletari F, Ahmadi SA, Kroll C, Plate A, Rozanski V, et al. (2016) Hough$\mathrm{cnn}$ : deep learning for segmentation of deep brain regions in MRI and ultrasound. Computer Vision \& Image Understanding.

6. Razzak MI, Naz S, Zaib A (2017) Deep learning for medical image processing: overview, challenges and the future. 
7. Litjens G, Kooi T, Bejnordi BE, Aaa S, Ciompi F, et al. (2017) A survey on deep learning in medical image analysis. Med Image Anal 42(9): 60-88.
8. Chen B, Polatkan G, Sapiro G, Blei D, Dunson D, et al. (2013) Deep learning with hierarchical convolutional factor analysis. IEEE Trans Pattern Anal Mach Intell 35(8): 1887-1901.

\section{Your next submission with JuniperPublishers will reach you the below assets}

- Quality Editorial service

- Swift Peer Review

- Reprints availability

- E-prints Service

- Manuscript Podcast for convenient understanding

- Global attainment for your research

- Manuscript accessibility in different formats

( Pdf, E-pub, Full Text, audio)

- Unceasing customer service

Track the below URL for one-step submission https://juniperpublishers.com/online-submission.php 